\title{
Reflets
}

Revue ontaroise d'intervention sociale et communautaire

\section{Rapports du Réseau des services de santé en français de l’Est de l'Ontario}

\section{Madeleine Dubois}

Volume 6, numéro 2, automne 2000

Problèmes sociaux en Ontario français

URI : https://id.erudit.org/iderudit/026331ar

DOI : https://doi.org/10.7202/026331ar

Aller au sommaire du numéro

Éditeur(s)

Reflets : Revue ontaroise d'intervention sociale et communautaire

ISSN

1203-4576 (imprimé)

1712-8498 (numérique)

Découvrir la revue

Citer ce compte rendu

Dubois, M. (2000). Compte rendu de [Rapports du Réseau des services de santé en français de l'Est de l'Ontario]. Reflets, 6(2), 264-268.

https://doi.org/10.7202/026331ar

Tous droits réservés (C) Reflets : Revue ontaroise d'intervention sociale et communautaire, 2000
Ce document est protégé par la loi sur le droit d'auteur. L'utilisation des services d'Érudit (y compris la reproduction) est assujettie à sa politique d'utilisation que vous pouvez consulter en ligne.

https://apropos.erudit.org/fr/usagers/politique-dutilisation/ 


\section{Rapports du Réseau des services de santé en français de l'Est de l'Ontario}

- Besoins et lacunes en matière de services de santé en français dans l'Est ontarien

- Les services de santé en français dans l'Est ontarien :besoins en formation de professionnels

Lu par Madeleine Dubois, École de service social, Université d'Ottawa

Créé en 1998 suite à une recommandation de la Commission de restructuration des services de santé de l'Ontario, le Réseau des services de santé en français de l'Est de l'Ontario a pour but d'" assurer aux francophones de l'Est de l'Ontario l'accès en français à toute la gamme de soins et de services de santé de qualité offerts par les établissements hospitaliers et autres organismes de santé et de fournir à ceux-ci un cadre de concertation pour leur permettre de satisfaire à leurs obligations de répondre aux besoins de la communauté francophone" (Lalonde $2000: 10)$. Pour être en mesure de répondre à ces objectifs, le Réseau a commandé deux études : la première a fait l'objet d'un rapport intitulé Besoins et lacunes en matière de services de santé en français dans l'Est ontarien, publié en septembre 1999; la deuxième, intitulée Les services de santé en français dans l'Est ontarien : besoins en formation de professionnels, a été publiée en avril 2000.

Les comptes rendus de ces deux rapports de recherche que nous présentons ici sont tirés du bulletin du Réseau intitulé Les Nouvelles du Réseau, qui est publié trois fois par année. 


\section{Besoins et lacunes en matière de services de santé en français dans l'Est ontarien}

À l'automne 1998, le Réseau des services de santé en français de l'Est de l'Ontario commandait deux études préparatoires à l'identification des besoins et des lacunes en matière de services de santé en français dans la région. La première consistait en un inventaire des services de santé disponibles sur tout le territoire, tandis que l'autre se voulait une recension des principales recommandations relatives aux services de santé en français contenues dans divers rapports publiés au cours des dix dernières années. Une série de consultations a ensuite été entamée au printemps 1999 dans le but de valider les renseignements présentés dans ces deux études. Ainsi, des personnes et groupes clés représentant les différents secteurs de la santé ont pu commenter la pertinence des recommandations antérieures recensées. Les consultations ont également été l'occasion d'identifier de nouveaux besoins ou lacunes dans le système actuel et de définir les actions concrètes pouvant être entreprises par le Réseau.

Le rapport intitulé Besoins et lacunes en matière de services de santé en français dans l'Est ontarien, qui rend compte des résultats de cette démarche, contient des données utiles relativement aux services de santé disponibles en français sur le territoire en question. Ces données sont présentées sous cinq catégories, soit : les soins hospitaliers, la santé communautaire, la santé publique, la santé mentale et la toxicomanie et les soins de longue durée. Le rapport contient également la liste des recommandations recensées dans les rapports antérieurs, ainsi que de nouveaux besoins et lacunes identifiés lors des consultations et ce, pour chacun des secteurs de la santé et pour chacune des régions suivantes: Ottawa-Carleton, Renfrew, Stormont, Dundas et Glengarry ainsi que PrescottRussell. L'exercice a aussi permis de cibler des actions prioritaires pour chacun des secteurs de la santé énoncés ci-dessus. Parmi celles-ci, notons :l'identification de modèles de prestation pouvant répondre aux besoins des francophones de l'est d'Ottawa-Carleton 
en matière de santé communautaire; l'élaboration d'un tableau complet des services francophones en santé mentale dans l'Est de l'Ontario et des lacunes à combler au sein de ceux-ci; et finalement, le travail à entreprendre avec les organismes francophones offrant des services de répit et des programmes de jour dans le but d'identifier les défis qu'ils traversent et les solutions possibles.

Les recommandations de ce rapport ont donné lieu à l'élaboration d'un plan de travail détaillé axé sur des stratégies et des mécanismes de mise en oeuvre, sur l'identification des ressources nécessaires et des échéanciers à respecter.

\section{Les services de santé en français dans l'Est ontarien : besoins en formation de professionnels}

Le 11 avril 2000,le Réseau rend public le rapport de la deuxième étude d'envergure qu'il avait entreprise au printemps 1999, dans le but d'évaluer plus précisément la question de la formation en français et des besoins de main-d'œuvre francophone dans le secteur de la santé. Cette recherche a été réalisée par Rolande Faucher, sous la direction d'un groupe de travail présidé par le $\mathrm{D}^{\text {re }}$ Jeanne Drouin de la Faculté de médecine de l'Université d'Ottawa et composé de représentants des différents secteurs et régions. Menée auprès d'établissements d'enseignement postsecondaire ainsi que d'établissements et d'organismes de santé de l'Est de l'Ontario, l'étude répertorie les programmes de formation en santé offerts en français en Ontario et identifie les domaines où il $\mathrm{y}$ a pénurie de professionnels de la santé francophones dans l'Est ontarien dans les secteurs des soins hospitaliers, de la santé mentale et de la toxicomanie, de la santé communautaire, de la santé publique et des soins de longue durée.

Le rapport présente un portrait des besoins de formation en santé en français et de professionnels de la santé francophones tel que dépeint par les quelque 80 personnes interviewées. Il se divise en deux volets : la formation et la main-d'œuvre. 
Le volet formation trace le profil de chacun des programmes collégiaux et universitaires en santé offerts en français en Ontario. Il présente les particularités de chacun, les enjeux auxquels ils sont confrontés et les projets en développement. Il relate les principales difficultés rencontrées par les établissements d'enseignement dans le recrutement et la rétention des étudiants et des professeurs ainsi que les stratégies gagnantes utilisées par certains. Cette section du rapport contient également une partie qui traite brièvement des programmes en santé qui sont offerts uniquement en anglais en Ontario. Plusieurs pistes de réflexion ont été relevées pour faciliter l'analyse contextuelle du développement de la formation en français dans les professions de la santé. Certains des éléments retenus touchent à l'interdisciplinarité, aux technologies de l'information et de la communication, à l'absence de certains programmes clés en français et au manque d'outils pédagogiques en français.

Par ailleurs, le volet main-d'œuvre présente les renseignements recueillis auprès d'établissements et d'organismes de santé offrant des services en français en mettant l'accent sur les thèmes suivants : la situation des services en français, le recrutement de professionnels francophones, les stratégies de rétention, les pénuries identifiées, les besoins actuels et futurs de professionnels francophones, l'aide requise et les besoins en matière de formation. Cette section du rapport permet de constater que les directives de la Commission de restructuration pour la région ont contribué à façonner le portrait d'un système de santé transformé par l'ampleur des changements, mais empreint d'incertitude quant au développement des services en français. Sur le plan du recrutement, on doit redoubler d'efforts et d'ingéniosité pour attirer et retenir les professionnels et on note par ailleurs une grande utilisation de mesures incitatives aux niveaux financier et humain. De plus, il appert qu'un milieu de travail à prédominance française et le caractère permanent des postes soient des éléments déterminants dans l'explication du faible taux de roulement enregistré par certains employeurs.

Les constatations faites dans le cadre des deux volets de l'étude ont mené à l'élaboration de six grandes conclusions qui ont à 
leur tour inspiré la formulation d'une trentaine de recommandations. Ces dernières ont été regroupées sous sept grands thèmes : le besoin d'appui au développement des services en français; la promotion des carrières en santé; le cheminement des professionnels francophones de la santé; le recrutement et la rétention des professionnels francophones; la formation en français des professionnels de la santé; les recommandations d'ordre général et les recommandations pour des études futures. Depuis le lancement du rapport, le Réseau a déjà élaboré un plan d'action afin d'assurer un suivi aux différentes recommandations de l'étude et organisé plusieurs rencontres avec les différents partenaires concernés.

En conclusion, quoique ces deux études s'appuient sur des données de recherche spécifiques à l'Est ontarien, la problématique dont il est question concerne néanmoins l'ensemble des francophones de l'Ontario. Les recommandations et les conclusions des études offrent des pistes de réflexion pertinentes pour la prestation des services de santé en français en Ontario et il serait donc utile que les rapports du Réseau soient diffusés au-delà du contexte géographique est ontarien.

Les personnes qui désirent obtenir un exemplaire du rapport peuvent communiquer avec le Réseau à l'adresse suivante :

1173, chemin Cyrville, bureau 300

Gloucester, ON K1J 7S6

Téléphone : (613) 747-7431

Télécopieur : (613) 747-2907

Courriel : reseau@rssfe.on.ca

Site Web : http ://www.rssfe.on.ca

\section{Référence bibliographique}

LALONDE, Jocelyne (2000). "Le réseau des services en français de l'Est de l'Ontario ", Bulletin, Section de l'Est: L'Association des travailleuses et travailleurs sociaux de l'Ontario, 26:2, 9-11. 